\title{
IWKNN: An Effective Bluetooth Positioning Method Based on Isomap and WKNN
}

\author{
Qi Wang, Yingying Feng, Xiangde Zhang, Yanrui Sun, and Xiaojun Lu \\ Department of Mathematics, Northeastern University, Shenyang, Liaoning Province, China \\ Correspondence should be addressed to Xiaojun Lu; luxiaojun0625@sina.com
}

Received 2 July 2016; Revised 21 September 2016; Accepted 29 September 2016

Academic Editor: Ruay-Shiung Chang

Copyright (C) 2016 Qi Wang et al. This is an open access article distributed under the Creative Commons Attribution License, which permits unrestricted use, distribution, and reproduction in any medium, provided the original work is properly cited.

\begin{abstract}
Recently, Bluetooth-based indoor positioning has become a hot research topic. However, the instability of Bluetooth RSSI (Received Signal Strength Indicator) promotes a huge challenge in localization accuracy. To improve the localization accuracy, this paper measures the distance of RSSI vectors on their low-dimensional manifold and proposes a novel positioning method IWKNN (Isomap-based Weighted $K$-Nearest Neighbor). The proposed method firstly uses Isomap to generate low-dimensional embedding for RSSI vectors. Then, the distance of two given RSSI vectors is measured by Euclidean distance of their low-dimensional embeddings. Finally, the position is calculated by WKNN. Experiment indicates that the proposed approach is more robust and accurate.
\end{abstract}

\section{Introduction}

Positioning is a basic requirement in people's daily life. It is the basis of LBS (Location-Based Service) [1,2]. Generally, positioning problems could be classified as outdoor and indoor. The outdoor positioning mainly uses GPS (Global Positioning System), GPRS (General Packet Radio Service), and so forth, while the indoor positioning mostly utilizes short range signals, such as Wi-Fi (Wireless-Fidelity) $[3,4]$ and Bluetooth [5-7]. And the testing position is determined based on the received signals. These positioning technologies have been widely used in different kinds of applications.

Beacon is a commonly used Bluetooth signal source. Figure 1 shows a picture of beacon. It is small and low-power Bluetooth dissipation equipment. It can work for even two years just with a fastener battery. So beacon can be placed without too many restrictions, having broad prospects of application.

This paper aims to improve the accuracy of Bluetoothbased indoor positioning, when beacons are preplaced at some given places in a room. A smart device is used to receive these signals. Then the position of device can be calculated based on received Bluetooth RSSI data.

Classical positioning methods could be classified as two types. One is function-based model. This kind of method is mostly developed based on signal propagation function $[8,9]$, for example, trilateration algorithm $[10,11]$ and IoT (Internet of Things) [12]. This kind of model requires an estimated signal propagation function. If the positions of emitters are given, then any testing location could be calculated based on RSSI data and signal propagation function. This kind of method does not need to store the vast RSSI data of reference positions. The localization accuracy relies on the measurement error of signal propagation function. The less the error is, the more accurate the localization is. Because the signal intensity of beacon is instable, the error of signal propagation model would be relatively large. So it is inaccurate to localize a Bluetooth receiver by this kind of method.

The other kind of method could be described as classification algorithm, which is built up based on position fingerprint. Figure 2 illustrates general positioning process based on fingerprint. Lemic et al. [13] and Jaffre et al. [14] determine the final position by KNN (K-Nearest Neighbor). They find the nearest $K$ reference positions to calculate unknown position according to Euclidean distance of RSSI vectors. Caso et al. [15] develop WKNN (Weighted $K$-Nearest Neighbor) for 3D positioning by weighting each nearest neighbor. Besides this, Shin et al. [16] propose EWKNN (Enhanced Weighted $K$-Nearest Neighbor) by making parameter $K$ variable to improve the positioning performance. 


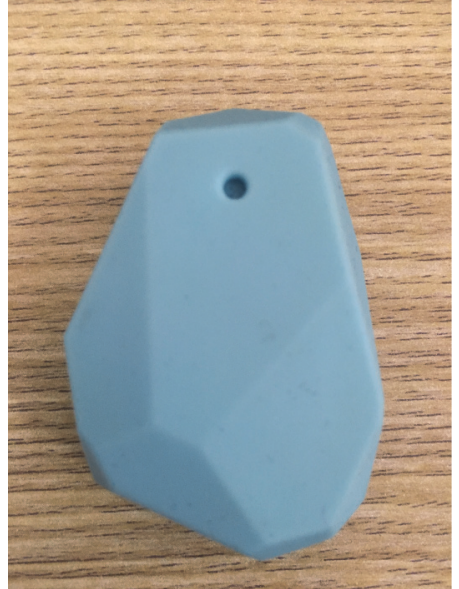

Figure 1: A picture of beacon.

Cheng et al. [17] introduce SVM (Support Vector Machine) to classify the RSSI vectors. Mu et al. [18] classify the RSSI data by ANN (Artificial Neural Network). Zhe [19] develops a positioning method based on Bayes method. He uses more flexible models to estimate distributions of beacon signals. Fras et al. [20] proposed a new method that combines Bayes and WKNN. Alfakih et al. [21] develop a positioning algorithm based on GMM (Gaussian Mixture Model). They calculate the probability that the testing RSSI belongs to each reference RSSI data. The final location is calculated by sum of weighted coordinates of reference locations. Different from Bayes method, GMM is to calculate the probability based on Gaussian Mixture function.

Kim et al. [22] and Thuong et al. [23] estimate the positioning performance for KNN algorithm. Jaffre et al. [14] compare KNN and Bayes positioning method with several different distance measurements and value $K$. Experimental result states that KNN and Euclidean distance can get the smallest mean positioning error when $K=4$. Zhang et al. [24] use beacons as emitter and compare the positioning performance of ANN, SVM, and improved KNN. The paper reports that the improved KNN algorithm has the highest positioning accuracy.

Because of the instability of Bluetooth, received RSSI vectors have a large variety. This means that RSSI vectors of faraway positions may have smaller Euclidean distances than that of neighborhood. It leads to choosing a faraway reference position as candidate location sometimes. This enlarges the localization error of Euclidean distance based methods, such as KNN and WKNN. So just using Euclidean distance to measure similarity of RSSI vectors is not accurate. Bluetooth positioning requires a more robust distance measurement method.

Manifold learning [25] finds the low-dimensional embedding for high-dimensional data. Researchers have proposed different algorithms for manifold learning, such as MDS (Multidimensional Scaling) [26], LLE (Locally Linear Embedding) [27], and Isomap [28]. The geodesic distance is a useful distance metric of Isomap in manifold learning. It can be used to measure the similarity of high-dimensional vectors. Figure 3 illustrates the comparison of Euclidian and geodesic distances.

We consider Bluetooth indoor positioning as a highdimensional data matching problem. Calculate low-dimensional embeddings for the training and testing RSSI data. Use the Euclidian distance of low-dimensional embeddings to measure the approaching extent of two given RSSI vectors. The final location is calculated by WKNN. Figure 4 demonstrates the flow chart of proposed method.

Figure 5 illustrates the comparison of positioning results of WKNN and proposed IWKNN. Figure 5(a) gives a localization illustration by WKNN based on Euclidian distance, while Figure 5(b) shows a positioning process by proposed IWKNN. In Figure 5, the red rectangles are real testing positions. The pink dots are selected $K$ nearest training positions $(K=4)$. The green hollow dots are obtained final position. Figure 5 shows that the proposed method can select more proper nearest positions than WKNN, achieving a better localization.

This paper is organized as follows: Section 2 introduces the mathematical model of indoor positioning problem. Section 3 illustrates the proposed localization algorithm based on Isomap and WKNN. Section 4 shows the experimental result and analysis. Section 5 concludes the whole paper.

\section{The Mathematical Model of Bluetooth Positioning}

This section presents the mathematical model of Bluetooth positioning problem.

Here, let us consider a general Bluetooth positioning problem. We establish a rectangular coordinate system in a region. $m$ beacons are distributed evenly in this area. Then a cell phone in this region would receive and measure RSSIs from all beacons. They can be grouped as a RSSI vector. The Bluetooth positioning problem is to determine the coordinate of cell phone in the rectangular coordinate system.

Suppose there are total $n$ reference positions. Administrator should record Bluetooth RSSI at each reference position for a period of time. When a user is standing at some place in this area, he can measure the Bluetooth RSSI by cell phone or other equipment. Then the coordinate of person can be calculated based on the newly measured and recorded RSSI data.

Let $x_{i}$ represent the coordinate of $i$ th reference location; $x_{i}=\left[a_{i}, b_{i}\right], i=1, \ldots, n \cdot \mathrm{rssi}_{i j}^{t}$ is the RSSI received from the $j$ th beacon at the $i$ th reference position at time $t . \alpha_{i}^{t}$ is a vector composed of RSSI on the $i$ th reference location from different beacons at time $t ; \alpha_{i}^{t}=\left[\mathrm{rssi}_{i 1}^{t}, \mathrm{rssi}_{i 2}^{t}, \ldots, \mathrm{rssi}_{i m}^{t}\right]^{T}$, $i=1, \ldots, n$. Here, $m$ is the number of beacons. $n$ is the number of reference locations.

If the RSSI data are separately received at time $t=$ $t_{1}, t_{2}, \ldots, t_{l}$, then the matrix of RSSI data for $i$ th reference locations could be written as

$$
\left[\alpha_{i}^{t_{1}}, \alpha_{i}^{t_{2}}, \ldots, \alpha_{i}^{t_{l}}\right]=\left(\begin{array}{cccc}
\operatorname{rssi}_{i 1}^{t_{1}} & \operatorname{rssi}_{i 1}^{t_{2}} & \cdots & \operatorname{rssi}_{i 1}^{t_{l}} \\
\operatorname{rssi}_{i 2}^{t_{1}} & \operatorname{rssi}_{i 2}^{t_{2}} & \cdots & \operatorname{rssi}_{i 2}^{t_{l}} \\
\vdots & \vdots & \ddots & \vdots \\
\operatorname{rssi}_{i m}^{t_{1}} & \operatorname{rssi}_{i m}^{t_{2}} & \cdots & \operatorname{rssi}_{i m}^{t_{l}}
\end{array}\right) .
$$




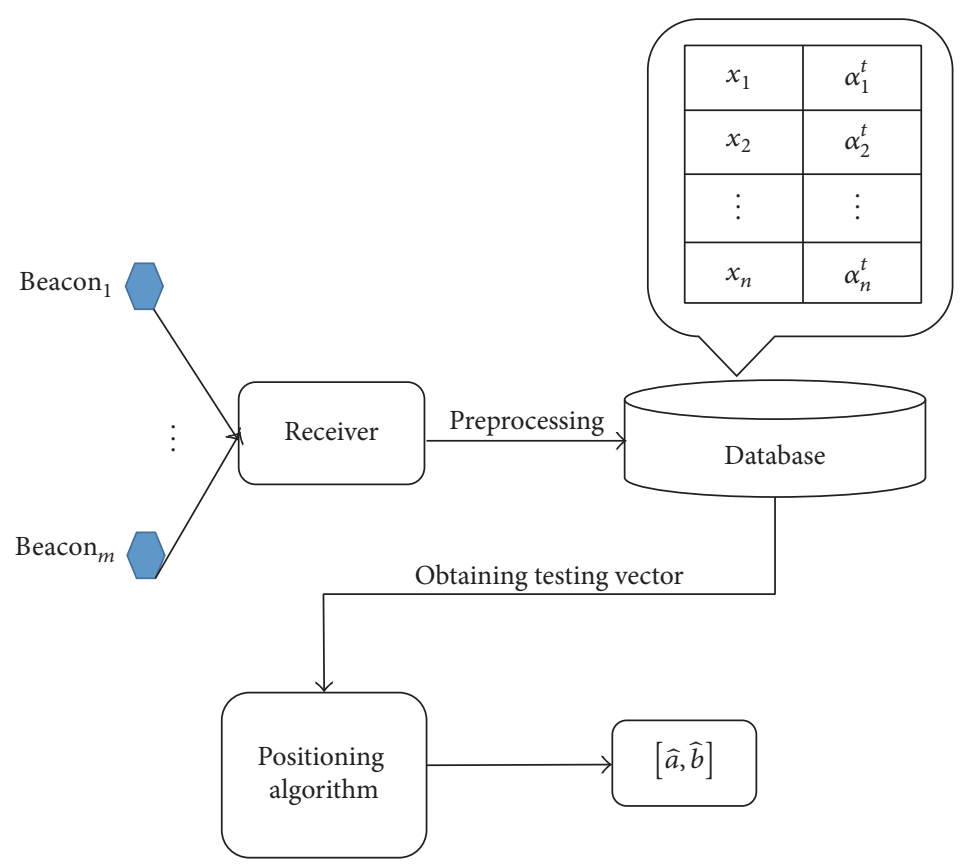

FIGURE 2: Positioning process based on fingerprint.

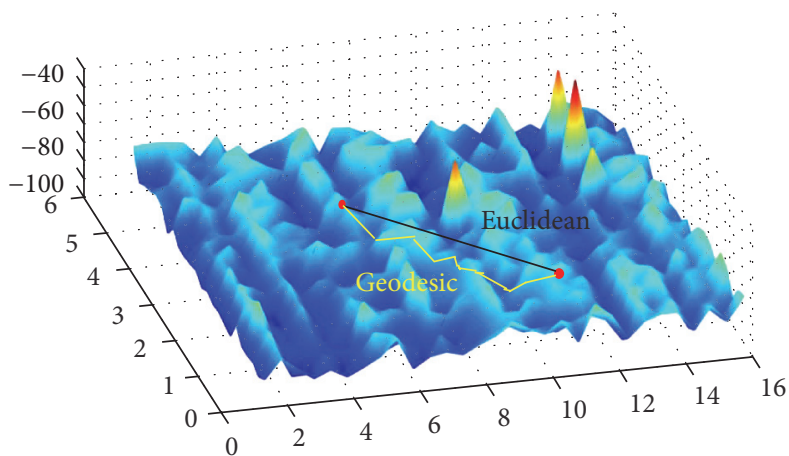

FIGURE 3: The difference of geodesic distance and Euclidean distance.

Let $\bar{\alpha}_{i}$ be the fingerprint of the $i$ th reference location; then

$$
\begin{aligned}
\bar{\alpha}_{i} & =\left[\overline{\operatorname{rssi}}_{i 1}, \overline{\operatorname{rssi}}_{i 2}, \ldots, \overline{\operatorname{rssi}}_{i m}\right]^{T} \\
& =\frac{1}{l}\left[\sum_{j=1}^{l} \operatorname{rssi}_{i 1}^{t_{j}}, \sum_{j=1}^{l} \operatorname{rssi}_{i 2} t_{j}, \ldots, \sum_{j=1}^{l} \operatorname{rssi}_{i 2}^{t_{j}}\right]^{T} .
\end{aligned}
$$

Here, $i=1,2, \ldots, n$.

For any unknown testing position $\vec{x}=[a, b]$, the received RSSI vector $\alpha_{x}$ at $\vec{x}$ could be written as

$$
\alpha_{x}=\left[\operatorname{rssi}_{x 1}, \operatorname{rssi}_{x 2}, \ldots, \mathrm{rssi}_{x m}\right]^{T} .
$$

Then the localization problem could be expressed by the following formula:

$$
[\widehat{a}, \widehat{b}]=T\left(\bar{\alpha}_{1}, \bar{\alpha}_{2}, \ldots, \bar{\alpha}_{n}, \alpha_{x}\right)
$$

Here, $T$ is the positioning function to be determined, which projects a RSSI vector to coordinate of position. This problem is generally solved by positioning fingerprint methods. These kinds of methods are mostly developed based on the Euclidean distance of RSSI vectors, such as $\operatorname{KNN}[13,14]$, WKNN [15], and EWKNN [16]. This paper develops a performance enhanced positioning method, which employs lowdimensional embedding distance to measure the similarity of RSSI vectors and use WKNN to determine unknown testing location.

\section{Proposed Method}

This section presents the proposed positioning method IWKNN. Firstly, the low-dimensional embeddings of reference and testing RSSI vectors are calculated by Isomap. Then, the unknown testing position is generated according to distances of low-dimensional embeddings by WKNN. 


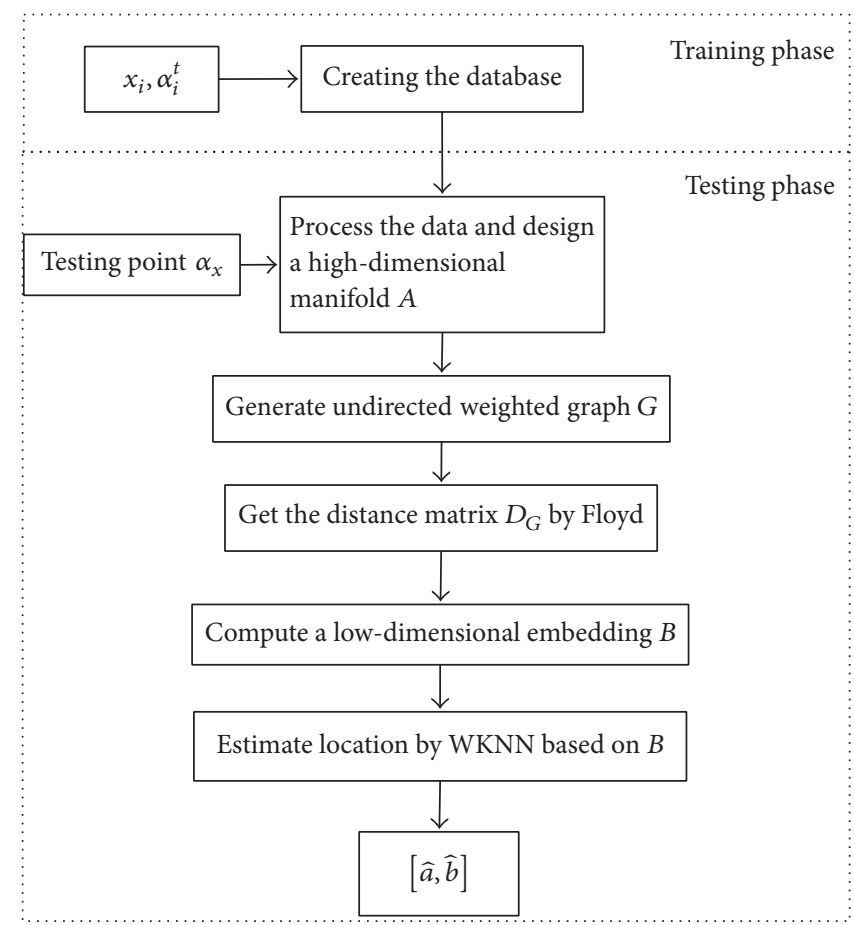

FIgURE 4: The block diagram of the proposed algorithm.

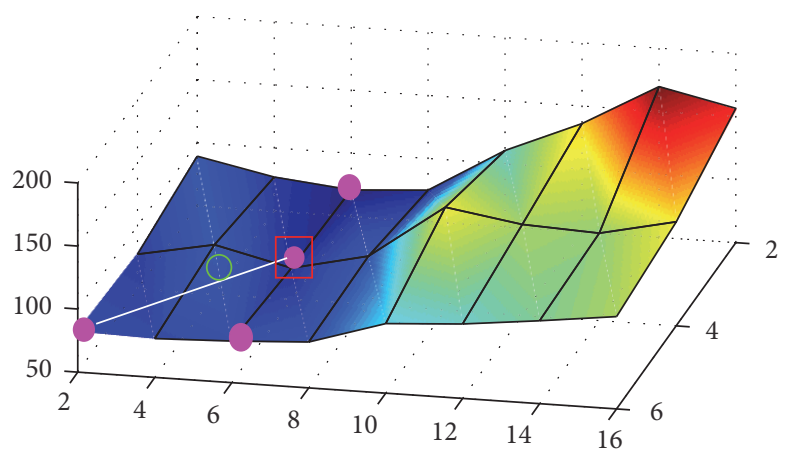

(a) Positioning by WKNN $(K=4)$

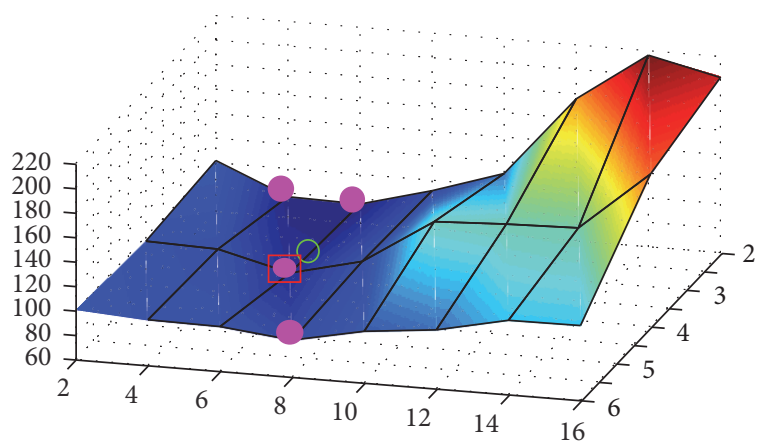

(b) Positioning by proposed IWKNN $(K=4)$

FIGURE 5: Positioning by WKNN and proposed IWKNN.

3.1. Overview. As an important method in manifold learning, Isomap can find the low-dimensional embedding for high-dimensional manifold. It can keep the neighborhood structure of high-dimensional manifold in a low-dimensional space.

Let $A$ be a set, which is composed of $(n+1)$ RSSI vectors.

$$
A=\left\{\bar{\alpha}_{i}, \alpha_{x} \mid i=1,2, \ldots, n\right\}
$$

$A \subset R^{m}$.

Suppose $B$ is a low-dimensional embedding of $A ; B=$ $\left\{\beta_{i}, \beta_{x} \mid i=1, \ldots, n\right\}$, where $B \subset R^{m^{\prime}}$ and $m^{\prime}<m$.

Let $f$ be a smooth embedding projection from $B$ to $A$, $f: B \rightarrow A$, satisfying $\alpha_{i}=f\left(\beta_{i}\right)$ (for all $i=1,2, \ldots, n$ ) and $\alpha_{x}=f\left(\beta_{x}\right)$.
Here, the low-dimensional embedding is calculated by Isomap. The distance of RSSI vectors is measured by Euclidian distance of their low-dimensional embeddings. Finally, the unknown testing position is calculated based on lowdimensional distances by WKNN.

3.2. Geodesic Distance of High-Dimensional Data. Assume all the elements in $A$ are from the same manifold. Ideally, any element in $A$ can be linearly expressed by its neighborhood points. We connect each point in $A$ to its $M$ nearest neighbors ( $M$ is a predetermined parameter). Then an undirected weighted graph is constructed. Thus, a distance matrix of this graph could be generated by Euclidean distance. The edge weight for given two points $\alpha_{i}$ and $\alpha_{j}$ is $d_{E}(i, j)=\left\|\alpha_{i}-\alpha_{j}\right\|$.

According to Isomap algorithm [28], the shortest distance of any two high-dimensional RSSI vectors is used as geodesic 


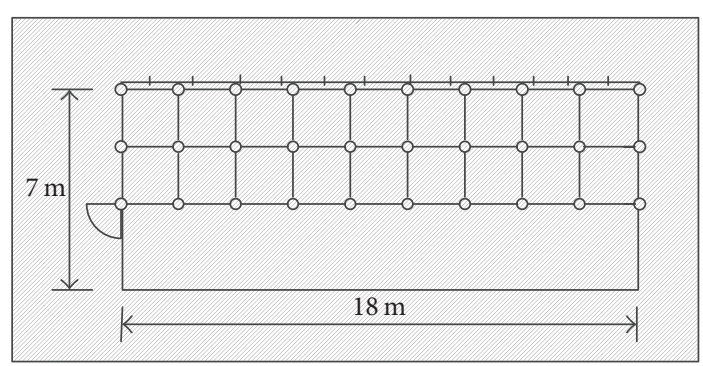

(a) The positions of beacons

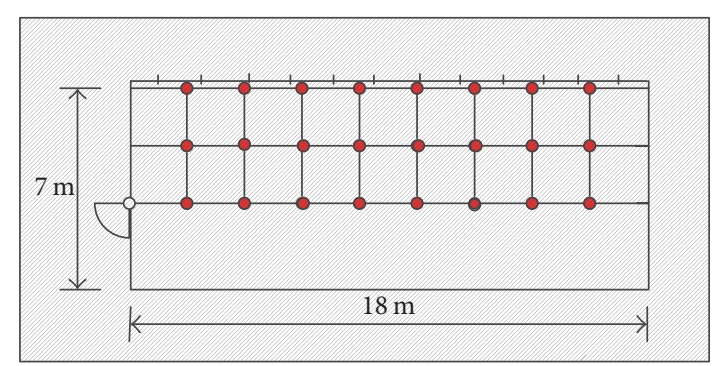

(b) The reference positions

Figure 6: The layout of beacons and reference positions.

distance. It can be calculated by Floyd's algorithm [28], which is shown in

$$
\begin{aligned}
& D_{G}=\left\{d_{G}(i, j)_{k}\right\} \\
& = \begin{cases}d_{E}(i, j) & k=0 \\
\min \left(d_{G}(i, j)_{k-1}, d_{G}(i, k)_{k-1}+d_{G}(k, j)_{k-1}\right) & k \geq 1 .\end{cases}
\end{aligned}
$$

Here, $k=0, \ldots, n+1$.

3.3. Low-Dimensional Embedding. Then a low-dimensional embedding could be calculated as follows [28]:

$$
\tau(D)=\frac{H S H}{-2}
$$

where $S_{i j}=\left(D_{i j}^{2}\right), H=I-(1 /(n+1)) E E^{T}, I$ is a unit matrix, and $E=[1,1, \ldots, 1]^{T}$.

Let $\lambda_{1}, \lambda_{2}, \ldots, \lambda_{m^{\prime}}$ be the largest $m^{\prime}$ eigenvalues of $\tau(D)$ (in descending order) and their corresponding eigenvectors be $u_{1}, u_{2}, \ldots, u_{m^{\prime}}$. Then all of the vectors in set $B$ could be obtained by [28]

$$
\begin{aligned}
& {\left[\beta_{1}, \beta_{2}, \ldots, \beta_{n}, \beta_{x}\right]} \\
& \quad=\left[\sqrt{\lambda_{1}} u_{1}, \sqrt{\lambda_{2}} u_{2}, \ldots, \sqrt{\lambda_{m^{\prime}}} u_{m^{\prime}}\right]^{T} .
\end{aligned}
$$

Then the low-dimensional embedding of $A=\left\{\bar{\alpha}_{1}, \bar{\alpha}_{2}\right.$, $\left.\ldots, \bar{\alpha}_{n}, \alpha_{x}\right\}$ is $B=\left\{\beta_{1}, \beta_{2}, \ldots, \beta_{n}, \beta_{x}\right\}$.

3.4. Distance of Low-Dimensional Embedding. The training low-dimensional fingerprint of the $i$ th reference point is $\left[\beta_{1}, \beta_{2}, \ldots, \beta_{n}\right]$. The testing low-dimensional vector is $\beta_{x}$. The distance of $\beta_{i}(i=1,2, \ldots, n)$ and $\beta_{x}$ is measured by Euclidian distance.

$$
d_{i}=\left\|\beta_{i}-\beta_{x}\right\|
$$

3.5. Positioning by WKNN. According to WKNN [15], let $d_{p_{1}}, d_{p_{2}}, \ldots, d_{p_{K}}$ stand for the $K$ minimum values in $\left\{d_{1}, d_{2}, \ldots, d_{n}\right\}$. Then the reference coordinates $\left[a_{p_{j}}, b_{p_{j}}\right], j=$ $1, \ldots, K$, are selected as candidate reference locations. Finally, the coordinate of testing position $[\widehat{a}, \widehat{b}]$ is estimated by the following formula [15]:

$$
[\widehat{a}, \hat{b}]=\frac{1}{\sum_{j=1}^{K} w_{j}} \sum_{j=1}^{K} w_{j}\left[a_{p_{j}}, b_{p_{j}}\right],
$$

where

$$
w_{j}=\frac{1}{d_{p_{j}}+c} .
$$

Here, $c$ is a relative small positive number.

\section{Experimental Result and Analysis}

4.1. Experimental Details. The experiment is carried out in a laboratory of Northeastern University. The laboratory room is $7 \times 18 \mathrm{~m}^{2}$. A $6 \times 18 \mathrm{~m}^{2}$ subarea is used as experimental area.

In our experiment, beacons are used to emit Bluetooth signals, whose parameters were adjusted referencing [5-7]. The emitting interval is set with $2 \mathrm{HZ}$ and power of $-8 \mathrm{db}$. Samsung Galaxy S3 is used to receive Bluetooth signals at a frequency of $1 \mathrm{~Hz}$.

Figure 6 shows the positions of beacons and reference locations. The hollow dots in Figure 6(a) show the layout of 30 beacons. They are placed evenly on the ceiling with mean distance of 2 meters. All the beacons are recorded with MAC address and positions. The solid dots in Figure 6(b) show the layout of 24 reference positions with mean distance of 2 meters on the ground.

To build a usable training fingerprint, RSSI data is recorded for 200 seconds at each reference position. And Kalman filter [29] is used to preprocess RSSI data.

Considering that a person may move fast with a cell phone, we just use one vector of RSSI data for positioning. In this paper, 74 testing positions are used in our experiment. These positions are distributed evenly with one-meter distance in the $6 \times 18 \mathrm{~m}^{2}$ experimental area.

4.2. Selection of $M$ and $m^{\prime}$. In Isomap algorithm, $M$ and $m^{\prime}$ are important parameters, which are the count of nearest neighbors and dimension of low-dimensional embedding. To select a proper $M$ and $m^{\prime}$, some experiments are carried out firstly. 
TABLE 1: The positioning errors with different $M$.

\begin{tabular}{lcccc}
\hline$M$ & Max error $(\mathrm{m})$ & Mean error $(\mathrm{m})$ & Min error $(\mathrm{m})$ & Std error $(\mathrm{m})$ \\
\hline 3 & 4.991 & 1.668 & 0.026 & 0.919 \\
4 & 5.389 & 1.657 & 0.230 & 1.006 \\
5 & 5.086 & 1.620 & 0.093 & 0.952 \\
6 & 3.851 & 1.796 & 0.473 & 0.907 \\
7 & 4.112 & 1.723 & 0.326 & 0.828 \\
8 & 4.278 & 1.910 & 0.335 & 0.953 \\
9 & 4.317 & 1.936 & 0.027 & 1.056 \\
10 & $\mathbf{3 . 4 0 0}$ & 1.865 & 0.078 & 0.818 \\
11 & 5.187 & 2.037 & 0.424 & 1.094 \\
12 & 5.806 & 1.983 & 0.389 & 1.148 \\
13 & 4.290 & 1.942 & 0.377 & 1.009 \\
14 & 5.388 & 1.938 & 0.292 & 0.971 \\
$\mathbf{1 5}$ & 3.814 & 1.672 & 0.278 & 0.791 \\
$\mathbf{1 6}$ & 3.815 & 1.592 & 0.303 & 0.775 \\
$\mathbf{1 7}$ & 3.528 & 1.635 & 0.334 & $\mathbf{0 . 7 2 6}$ \\
$\mathbf{1 8}$ & 4.516 & 1.732 & 0.297 & 0.841 \\
$\mathbf{1 9}$ & 3.602 & 1.635 & 0.331 & 0.864 \\
$\mathbf{2 0}$ & 3.605 & $\mathbf{1 . 5 1 6}$ & $\mathbf{0 . 0 0 1}$ & 0.782 \\
$\mathbf{2 1}$ & 3.600 & 1.618 & 0.117 & 0.776 \\
22 & 4.601 & 1.655 & 0.350 & 0.976 \\
23 & 4.590 & 1.622 & 0.345 & 0.934 \\
24 & 4.902 & 1.633 & 0.345 & 0.982 \\
\hline
\end{tabular}

TABLE 2: The mean positioning error with different $m^{\prime}$.

\begin{tabular}{lccccccc}
\hline$m^{\prime} / M$ & $\mathbf{1 5}$ & $\mathbf{1 6}$ & $\mathbf{1 7}$ & $\mathbf{1 8}$ & $\mathbf{1 9}$ & $\mathbf{2 0}$ & $\mathbf{2 1}$ \\
\hline 2 & 1.778 & 1.772 & 1.957 & 1.807 & 1.895 & 2.004 & 1.920 \\
3 & 1.856 & 1.710 & 1.811 & 1.735 & 1.680 & 1.801 & 1.712 \\
$\mathbf{4}$ & 1.770 & 1.744 & 1.687 & $\mathbf{1 . 6 6 3}$ & $\mathbf{1 . 6 0 1}$ & 1.573 & $\mathbf{1 . 5 2 9}$ \\
$\mathbf{5}$ & 1.672 & 1.592 & $\mathbf{1 . 6 3 5}$ & 1.732 & 1.635 & $\mathbf{1 . 5 1 6}$ & 1.618 \\
$\mathbf{6}$ & $\mathbf{1 . 6 2 5}$ & $\mathbf{1 . 5 2 3}$ & 1.712 & 1.736 & 1.657 & 1.597 & 1.617 \\
7 & 1.741 & 1.600 & 1.705 & 1.703 & 1.761 & 1.675 & 1.609 \\
8 & 1.751 & 1.585 & 1.703 & 1.783 & 1.614 & 1.731 & 1.594 \\
9 & 1.770 & 1.695 & 1.718 & 1.843 & 1.639 & 1.730 & 1.593 \\
10 & 1.778 & 1.686 & 1.666 & 1.825 & 1.672 & 1.682 & 1.606 \\
11 & 1.785 & 1.676 & 1.718 & 1.823 & 1.749 & 1.695 & 1.559 \\
12 & 1.764 & 1.618 & 1.722 & 1.776 & 1.665 & 1.646 & 1.584 \\
13 & 1.742 & 1.577 & 1.695 & 1.761 & 1.630 & 1.635 & 1.592 \\
14 & 1.736 & 1.633 & 1.697 & 1.755 & 1.653 & 1.675 & 1.615 \\
15 & 1.745 & 1.638 & 1.674 & 1.741 & 1.678 & 1.702 & 1.606 \\
\hline & & & & & & &
\end{tabular}

Table 1 shows the positioning errors with different $M$. As can be seen from the table, when $M$ is between 15 and 21 , the maximum and mean positioning errors are relatively small and stable. According to our experiment, the proposed method achieves minimum mean error when $M=20$.

Table 2 shows the mean positioning error with different $M$ and $m^{\prime} . M$ is assigned between 15 and 21. $m^{\prime}$ varies from 2 to 15 . In this table, we can find that when $m^{\prime}$ is between 4 and 6 , the mean positioning error is relatively small.

According to these experiments, we set $M=20$ and $m^{\prime}=$ 5 in this paper.

4.3. The Effectiveness of Isomap. The effectiveness of Isomap is firstly examined in our Bluetooth localization experiment. We compare the performance of WKNN and EWKNN [16] and their Isomap enhanced versions. WKNN is separately tested with $K=3,4, \ldots, 9$. And the threshold of EWKNN is mean of distance vector.

Figure 7 shows the error distribution of different methods. In Figure 7, the first-column figures illustrate the error distributions of original methods. The second column shows the error distribution of Isomap enhanced methods. The third column shows the differences of original and Isomap enhanced methods. From Figure 7, we can find that the error distribution of developed Isomap enhanced methods (IWKNN and IEWKNN) is more concentrated in the low error region than original methods (WKNN and EWKNN).

Figure 8 illustrates the cumulative probability of error distribution for different methods. The curves in these figures demonstrate that the Isomap enhanced methods (IWKNN and IEWKNN) achieve better performance than original methods (WKNN and EWKNN).

Finally, the performance of all tested methods is given in Table 3. According to this table, Isomap almost improves all of positioning indicators clearly. The best values of important indicators are given in bold font. The minimum of $\max$ error is 4.994, which is obtained by IWKNN with $K=9$. The minimum of mean error is 1.463 , which is obtained by IWKNN with $K=4$. The minimum of min error is 0.0005 , which is obtained by IWKNN with $K=3$ and IEWKNN. The minimum of std error is 0.674 , which is obtained by IWKNN with $K=7$. The maximum of "ratio of error less than 2 meters" is $78.96 \%$, which is obtained by IWKNN with $K=5$. This table supports the effectiveness of proposed Isomap enhanced positioning methods.

According to Table 3, we suggest $K=4$ or 5 for small mean positioning error and large "ratio of error less than 2 meters."

4.4. Comparison with Different Methods. In order to test the performance of proposed method more clearly, we compare the proposed IWKNN $(K=4)$ with six different positioning methods.

Figure 9 compares the performances of different methods. From this table, we can see that Bayes method has the best performance in the low error part, and the proposed IWKNN performs best in almost all the remaining part.

Table 4 illustrates the positioning error of different methods. According to this table, GMM, Bayes, and WKNNBayes's max errors are better than other methods. But the improved Bayes method proposed by Fras et al. [20] can generate small max, mean, and std errors. The proposed IWKNN has the minimal mean error and maximal "ratio of error less than 2 meters." 


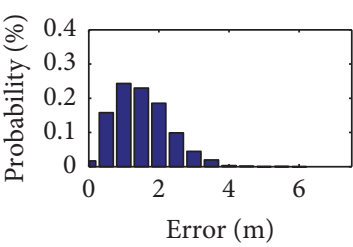

(a) $\operatorname{WKNN}(K=3)$

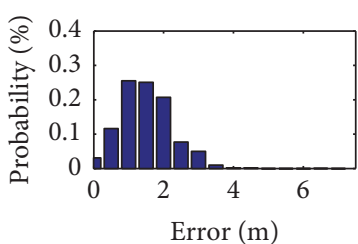

(d) $\operatorname{WKNN}(K=4)$

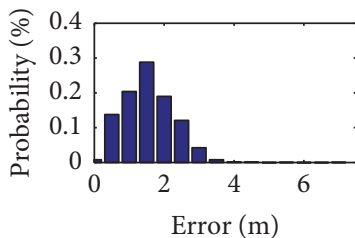

(g) WKNN $(K=5)$

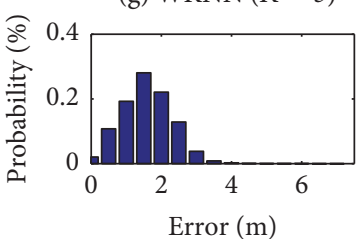

(j) $\operatorname{WKNN}(K=6)$

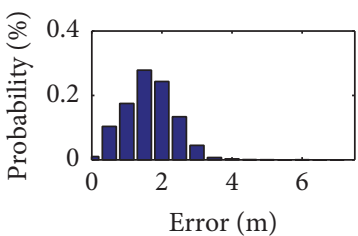

(m) WKNN $(K=7)$

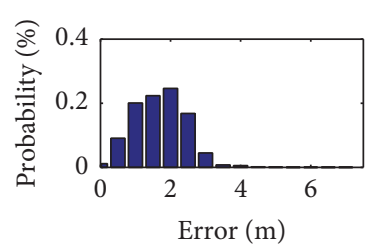

(p) WKNN $(K=8)$

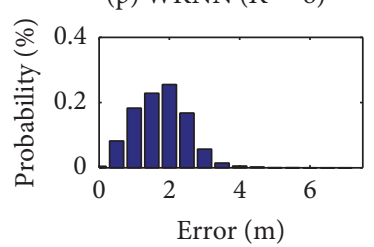

(s) WKNN $(K=9)$

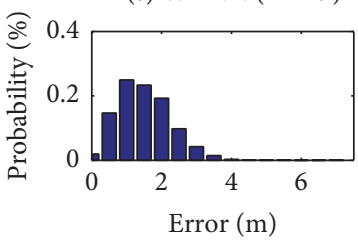

(v) EWKNN

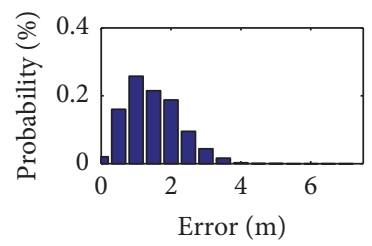

(b) IWKNN $(K=3)$

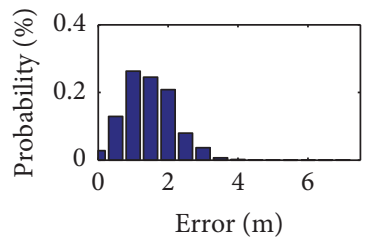

(e) IWKNN $(K=4)$

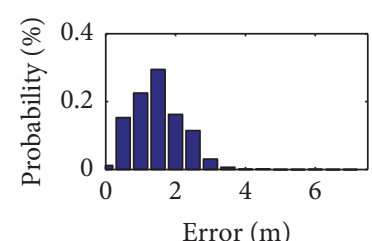

(h) IWKNN $(K=5)$

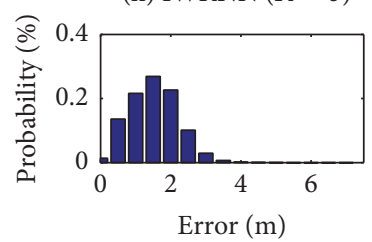

(k) IWKNN $(K=6)$

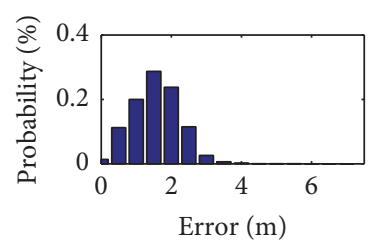

(n) $\operatorname{IWKNN}(K=7)$

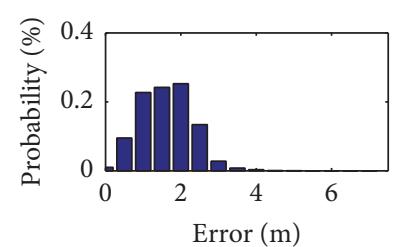

(q) $\operatorname{IWKNN}(K=8)$

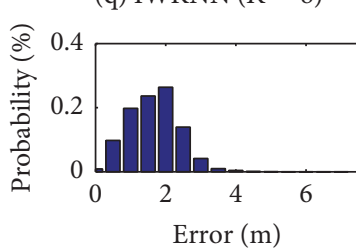

(t) IWKNN $(K=9)$

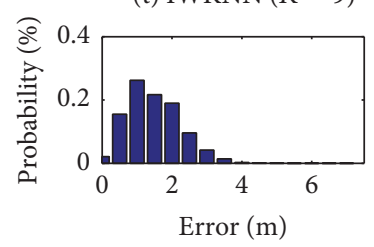

(w) IEWKNN (Isomap EWKN-N)

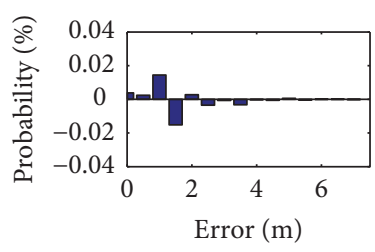

(c) (b)-(a)

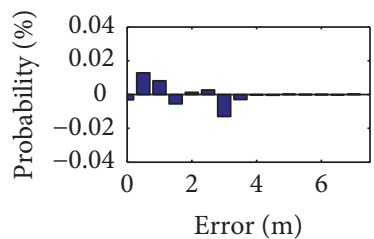

(f) (e)-(d)

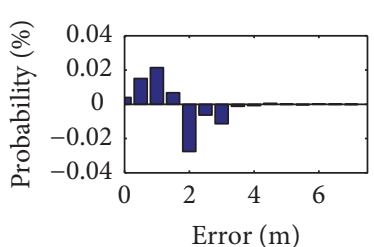

(i) (h) $-(\mathrm{g})$

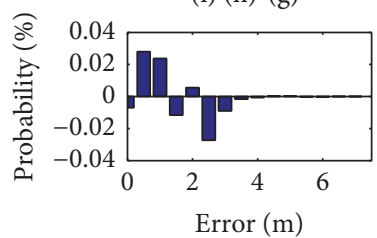

(l) (k)-(j)

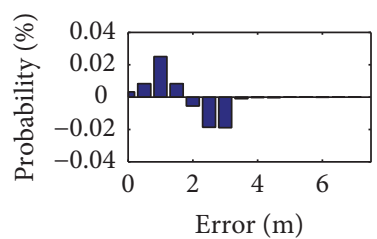

(o) (n)-(m)

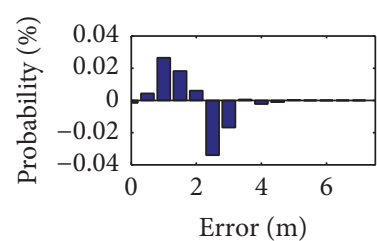

(r) (q)-(p)

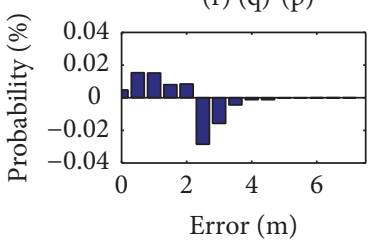

(u) $(\mathrm{t})-(\mathrm{s})$

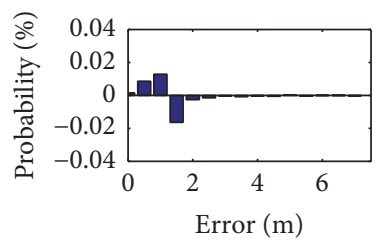

(x) (w)-(v)

FIGURE 7: Error distributions of original and Isomap enhanced methods. 


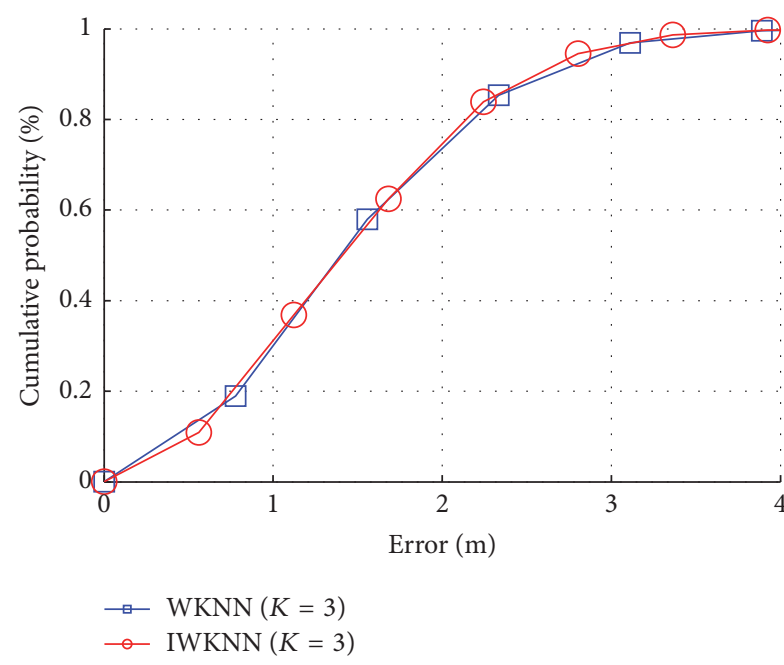

(a) WKNN and IWKNN $(K=3)$

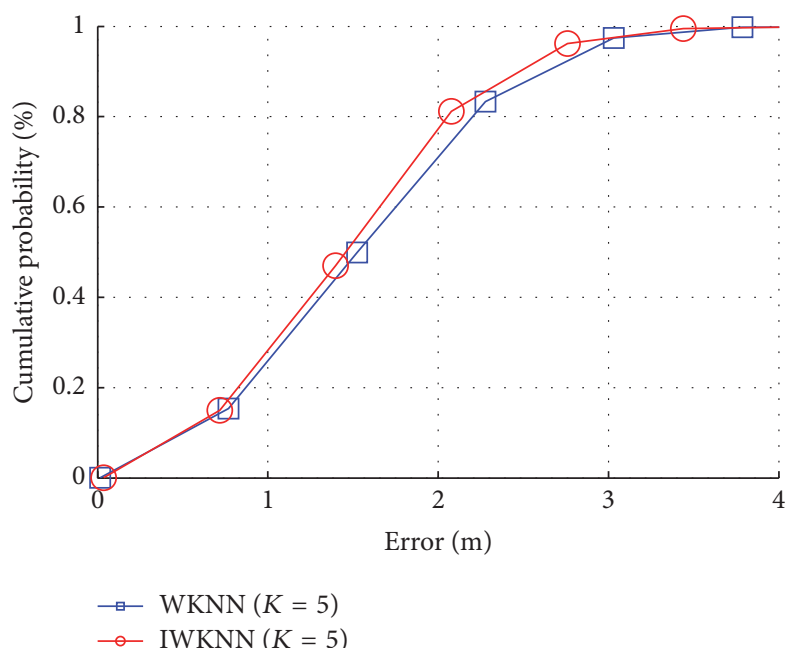

(c) WKNN and IWKNN $(K=5)$

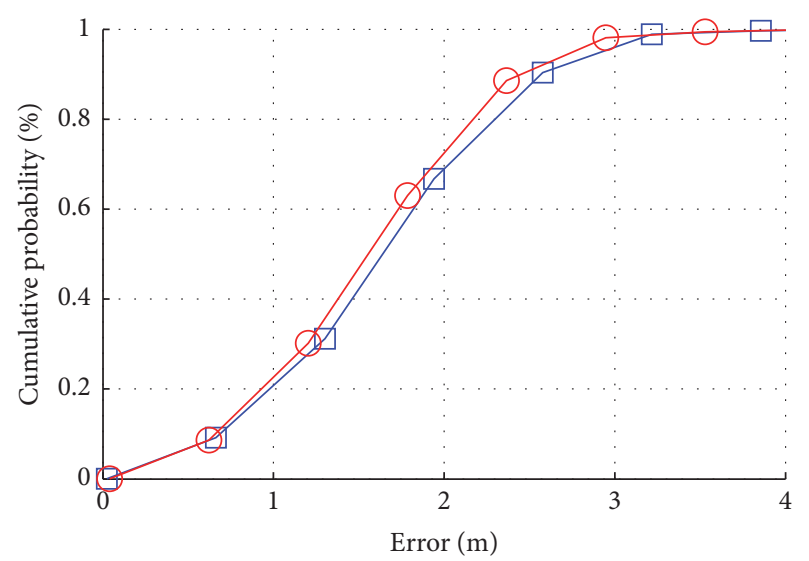

$\square \operatorname{WKNN}(K=7)$

$\multimap \operatorname{IWKNN}(K=7)$

(e) WKNN and IWKNN $(K=7)$

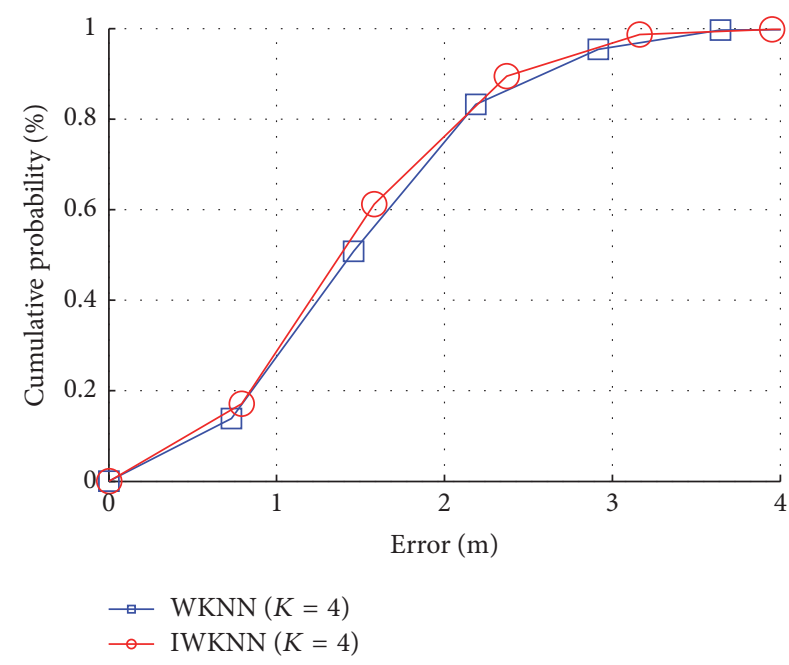

(b) WKNN and IWKNN $(K=4)$

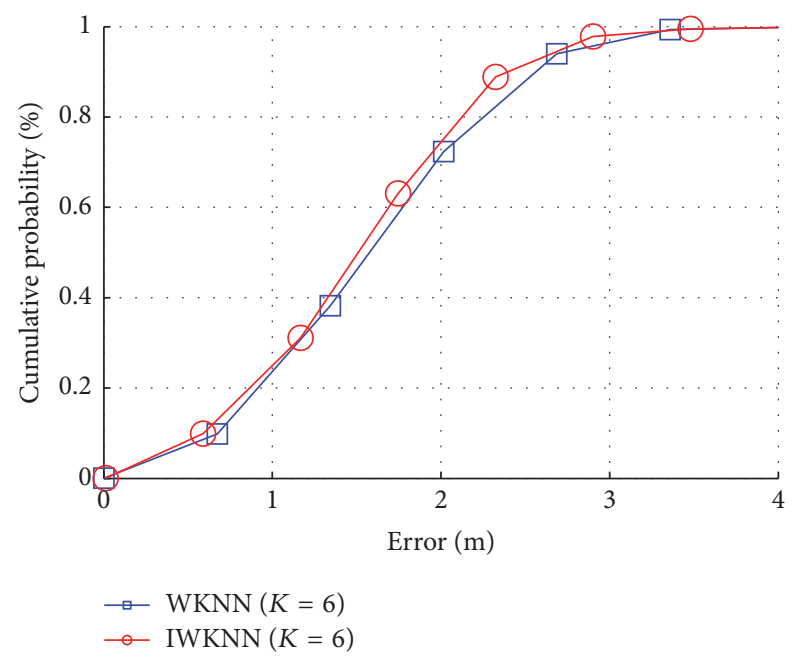

(d) WKNN and IWKNN $(K=6)$

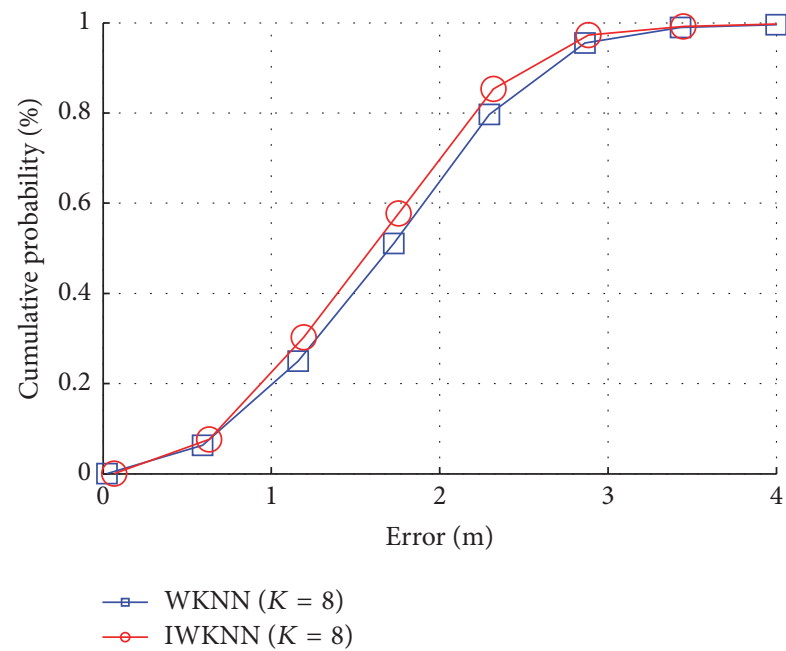

(f) WKNN and IWKNN $(K=8)$

Figure 8: Continued. 


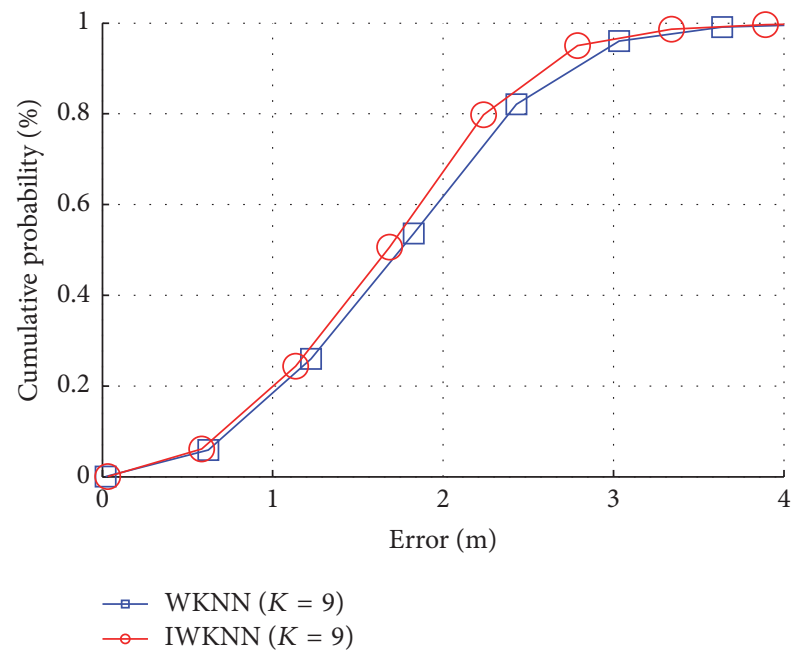

(g) WKNN and $\operatorname{IWKNN}(K=9)$

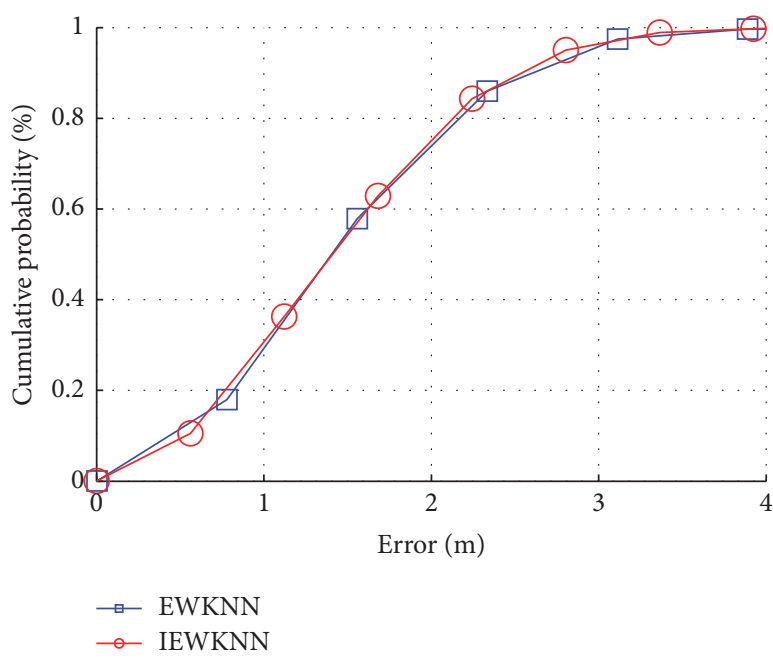

(h) EWKNN and IEWKNN

FIGURE 8: Cumulative error distribution of original and Isomap enhanced methods.

TABLE 3: The positioning error of original and Isomap enhanced methods.

\begin{tabular}{|c|c|c|c|c|c|}
\hline Method & Max error (m) & Mean error $(\mathrm{m})$ & Min error $(\mathrm{m})$ & Std error $(\mathrm{m})$ & $\leq 2 \mathrm{~m}(\%)$ \\
\hline WKNN $(K=3)$ & 7.000 & 1.505 & 0.0008 & 0.772 & $73.99 \%$ \\
\hline $\operatorname{IWKNN}(K=3)$ & 5.046 & 1.481 & 0.0005 & 0.758 & $75.58 \%$ \\
\hline WKNN $(K=4)$ & 6.558 & 1.508 & 0.001 & 0.732 & $74.49 \%$ \\
\hline $\operatorname{IWKNN}(K=4)$ & 7.110 & 1.463 & 0.002 & 0.710 & $76.05 \%$ \\
\hline WKNN $(K=5)$ & 6.803 & 1.541 & 0.014 & 0.712 & $76.12 \%$ \\
\hline IWKNN $(K=5)$ & 6.162 & 1.478 & 0.036 & 0.693 & $78.96 \%$ \\
\hline WKNN $(K=6)$ & 6.045 & 1.581 & 0.002 & 0.707 & $71.45 \%$ \\
\hline IWKNN $(K=6)$ & 5.216 & 1.512 & 0.011 & 0.680 & $77.08 \%$ \\
\hline WKNN $(K=7)$ & 5.774 & 1.631 & 0.023 & 0.703 & $69.18 \%$ \\
\hline IWKNN $(K=7)$ & 5.273 & 1.558 & 0.041 & 0.674 & $74.27 \%$ \\
\hline WKNN $(K=8)$ & 5.135 & 1.689 & 0.023 & 0.721 & $65.50 \%$ \\
\hline IWKNN $(K=8)$ & 5.138 & 1.604 & 0.066 & 0.683 & $70.77 \%$ \\
\hline WKNN $(K=9)$ & 5.447 & 1.749 & 0.019 & 0.739 & $61.51 \%$ \\
\hline IWKNN $(K=9)$ & 4.994 & 1.660 & 0.032 & 0.703 & $67.14 \%$ \\
\hline EWKNN & 7.000 & 1.502 & 0.002 & 0.756 & $74.26 \%$ \\
\hline IEWKNN & 5.046 & 1.477 & 0.0005 & 0.743 & $76.04 \%$ \\
\hline
\end{tabular}

TABLE 4: The positioning error of different methods.

\begin{tabular}{lccccc}
\hline Method & Max error $(\mathrm{m})$ & Mean error $(\mathrm{m})$ & Min error $(\mathrm{m})$ & Std error $(\mathrm{m})$ & $\leq 2 \mathrm{~m}(\%)$ \\
\hline Triangulation & 11.443 & 2.592 & 0.0288 & 1.616 & $41.80 \%$ \\
IoT [12] & 7.576 & 3.077 & 0.0331 & 1.482 & $27.72 \%$ \\
GMM [21] & 12.880 & 3.506 & $\mathbf{0}$ & 665 & 1.611 \\
Bayes & 14.318 & 2.391 & $\mathbf{0}$ & $\mathbf{0}$ & 4.116 \\
WKNN-Bayes [20] & 8.062 & 2.031 & 0.001 & 0.732 & $53.42 \%$ \\
WKNN & $\mathbf{6 . 5 5 8}$ & 1.508 & 0.002 & $\mathbf{0 . 7 1 0}$ & $74.49 \%$ \\
IWKNN & 7.110 & $\mathbf{1 . 4 6 3}$ & & $\mathbf{7 6 . 0 5} \%$ \\
\hline
\end{tabular}

\section{Conclusion}

This paper presents an Isomap enhanced localization method IWKNN. The proposed method combines Isomap and WKNN for Bluetooth positioning. Firstly, we calculate lowdimensional embeddings for RSSI data by Isomap. Then, the distance of different RSSI vectors is measured by Euclidean distance of these low-dimensional embeddings. Finally, the unknown testing position is calculated by WKNN. Experiment indicates the distances of low-dimensional embeddings are more robust than those of high-dimensional ones. And the proposed IWKNN is robust and effective.

The main contribution of this paper could be concluded as follows: (1) introduce Isomap to Bluetooth positioning 


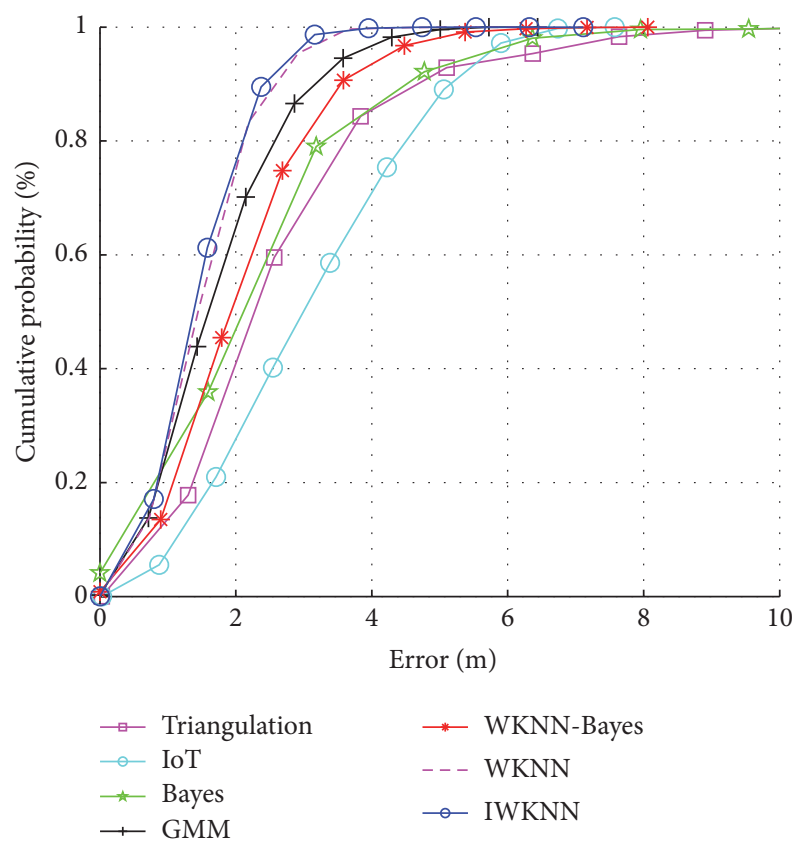

FIgURE 9: The cumulative error distribution of different methods.

problem; (2) use low-dimensional embeddings to represent high-dimensional RSSI data; (3) adopt distances of lowdimensional embeddings to measure the distance of RSSI vectors; (4) combine Isomap with WKNN for Bluetooth positioning.

\section{Disclosure}

The method presented in this paper has been applied for patent.

\section{Competing Interests}

The authors declare that they have no competing interests.

\section{References}

[1] A. Petrenko, A. Sizo, W. Qian et al., "Exploring mobility indoors: an application of sensor-based and GIS systems," Transactions in GIS, vol. 18, no. 3, pp. 351-369, 2014.

[2] W. Wang and R. Zhou, "Research on the anti-collision system of surface coal mine based on the highly accurate GPS location technology," in Proceedings of the 9th International Conference on Electronic Measurement and Instruments (ICEMI '09), pp. 3196-3199, Beijing, China, August 2009.

[3] A. Narzullaev and Y. Park, "Novel calibration algorithm for received signal strength based indoor real-time locating systems," AEU-International Journal of Electronics and Communications, vol. 67, no. 7, pp. 637-644, 2013.

[4] K. Kaemarungsi and P. Krishnamurthy, "Analysis of WLAN's received signal strength indication for indoor location fingerprinting," Pervasive and Mobile Computing, vol. 8, no. 2, pp. 292-316, 2012.
[5] R. Faragher and R. Harle, "Location fingerprinting with bluetooth low energy beacons," IEEE Journal on Selected Areas in Communications, vol. 33, no. 11, pp. 2418-2428, 2015.

[6] H. K. Fard, Y. Chen, and K. K. Son, "Indoor positioning of mobile devices with agile iBeacon deployment," in Proceedings of the 28th IEEE Canadian Conference on Electrical and Computer Engineering (CCECE '15), pp. 275-279, IEEE, Halifax, Canada, May 2015.

[7] F. Subhan, H. Hasbullah, A. Rozyyev, and S. T. Bakhsh, "Analysis of bluetooth signal parameters for indoor positioning systems," in Proceedings of the IEEE International Conference on Computer Information Science (ICCIS '12 ), vol. 2, pp. 784-789, Kuala Lumpur, Malaysia, June 2012.

[8] F. Palumbo, P. Barsocchi, S. Chessa, and J. C. Augusto, "A stigmergic approach to indoor localization using Bluetooth Low Energy beacons," in Proceedings of the 12th IEEE International Conference on Advanced Video and Signal Based Surveillance (AVSS '15), pp. 1-6, Karlsruhe, Germany, August 2015.

[9] T. Pulkkinen, J. Verwijnen, and P. Nurmi, "WiFi positioning with propagation-based calibration," in Proceedings of the 14th International Symposium on Information Processing in Sensor Networks (IPSN '15), pp. 366-367, Seattle, Wash, USA, April 2015.

[10] M. E. Rida, F. Liu, Y. Jadi, A. A. A. Algawhari, and A. Askourih, "Indoor location position based on bluetooth signal strength," in Proceedings of the 2nd International Conference on Information Science and Control Engineering (ICISCE '15), pp. 769-773, Shanghai, China, April 2015.

[11] S. Boonsriwai and A. Apavatjrut, "Indoor wifi localization on mobile devices," in Proceedings of the 10th IEEE International Conference on Electrical Engineering Electronics, Computer, Telecommunications and Information Technology (ECTI-CON '13), pp. 1-5, Krabi, Thailand, May 2013.

[12] H. N. Thi and K. N. T. Vu, "A positioning algorithm in the internet of things," in Proceedings of the 8th International Conference on Ubiquitous and Future Networks (ICUFN '16), pp. 594-598, Vienna, Austria, July 2016.

[13] F. Lemic, A. Behboodi, V. Handziski, and A. Wolisz, "Experimental decomposition of the performance of fingerprintingbased localization algorithms," in Proceedings of the 5th IEEE International Conference on Indoor Positioning and Indoor Navigation (IPIN '14), pp. 355-364, Busan, South Korea, October 2014.

[14] T. Jaffre, P.-M. Grigis, S. Papanastasiou, and E. Peytchev, "On the efficacy of WiFi indoor positioning in a practical setting," in Proceedings of the 18th IEEE Symposium on Computers and Communications (ISCC '13), pp. 699-704, July 2013.

[15] G. Caso, L. De Nardis, and M.-G. Di Benedetto, "Frequentist inference for WiFi fingerprinting 3D indoor positioning," in Proceedings of the IEEE International Conference on Communication Workshop (ICCW '15), pp. 809-814, IEEE, London, UK, June 2015.

[16] B. Shin, J. H. Lee, T. Lee, and H. S. Kim, "Enhanced weighted knearest neighbor algorithm for indoor wi-fi positioning systems," in Proceedings of the 8th International Conference on Computing Technology and Information Management (ICCM '12), vol. 2, pp. 574-577, IEEE, Berlin, Germany, 2012.

[17] J. Cheng, Y. Cai, Q. Zhang, J. Cheng, and C. Yan, "A new threedimensional indoor positioning mechanism based on wireless LAN," Mathematical Problems in Engineering, vol. 2014, Article ID 862347, 7 pages, 2014. 
[18] Z. Mu, X. Yubin, and M. Lin, "ANN indoor position determination based on area correlation in WLAN environment," in Proceedings of the International Conference on Wireless Communications and Signal Processing (WCSP '09), pp. 1-4, IEEE, Nanjing, China, November 2009.

[19] C. Zhe, "The research and implementation of indoor location positioning system based on low-power bluetooth and location fingerprint," 2014.

[20] M. Fras, K. Wasko, and T. Wierzowiecki, Personal Wi-Fi Based Indoor Localization of Mobile Devices in Active Environment, Springer, New York, NY, USA, 2016.

[21] M. Alfakih, M. Keche, and H. Benoudnine, "Gaussian mixture modeling for indoor positioning wifi systems," in Proceedings of the 3rd International Conference on Control, Engineering and Information Technology (CEIT '15), Tlemcen, Algeria, May 2015.

[22] J. Kim, M. Ji, J. il Jeon, S. Park, and Y. Cho, "K-NN based positioning performance estimation for fingerprinting localization," in Proceedings of the 8th International Conference on Ubiquitous and Future Networks (ICUFN '16), pp. 468-470, Vienna, Austria, July 2016.

[23] N. T. Thuong, H. T. Phong, D.-T. Do, P. V. Hieu, and D. T. Loc, "Android application for WiFi based indoor position: system design and performance analysis," in Proceedings of the International Conference on Information Networking (ICOIN '16), pp. 416-419, Kota Kinabalu, Malaysia, January 2016.

[24] L. Zhang, X. Liu, J. Song, C. Gurrin, and Z. Zhu, "A comprehensive study of bluetooth fingerprinting-based algorithms for localization," in Proceedings of the 27th International Conference on Advanced Information Networking and Applications Workshops (WAINA '13), pp. 300-305, Barcelona, Spain, March 2013.

[25] W. Zhan, G. Dai, and H. Liu, "Research overview of manifold learning algorithm," Advances in Information Sciences and Service Sciences, vol. 5, no. 1, pp. 58-67, 2013.

[26] I. Borg and P. J. Groenen, Modern Multidimensional Scaling: Theory and Applications, Springer Science \& Business Media, Berlin, Germany, 2005.

[27] S. T. Roweis and L. K. Saul, "Nonlinear dimensionality reduction by locally linear embedding," Science, vol. 290, no. 5500, pp. 2323-2326, 2000.

[28] J. B. Tenenbaum, V. De Silva, and J. C. Langford, "A global geometric framework for nonlinear dimensionality reduction," Science, vol. 290, no. 5500, pp. 2319-2323, 2000.

[29] G. Welch and G. Bishop, An Introduction to the Kalman Filter, University of North Carolina at Chapel Hill, Chapel Hill, NC, USA, 1995. 

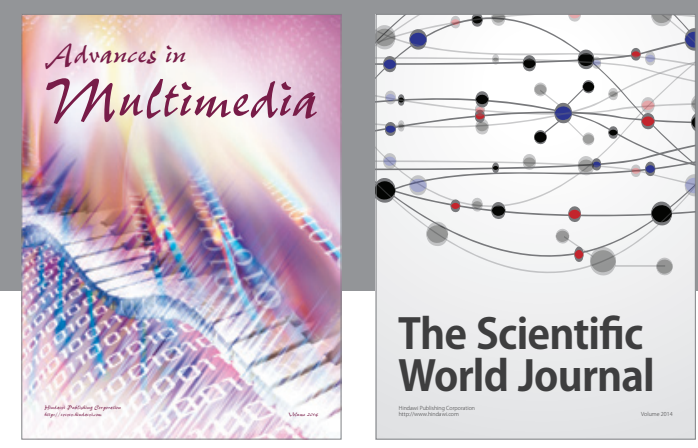

The Scientific World Journal
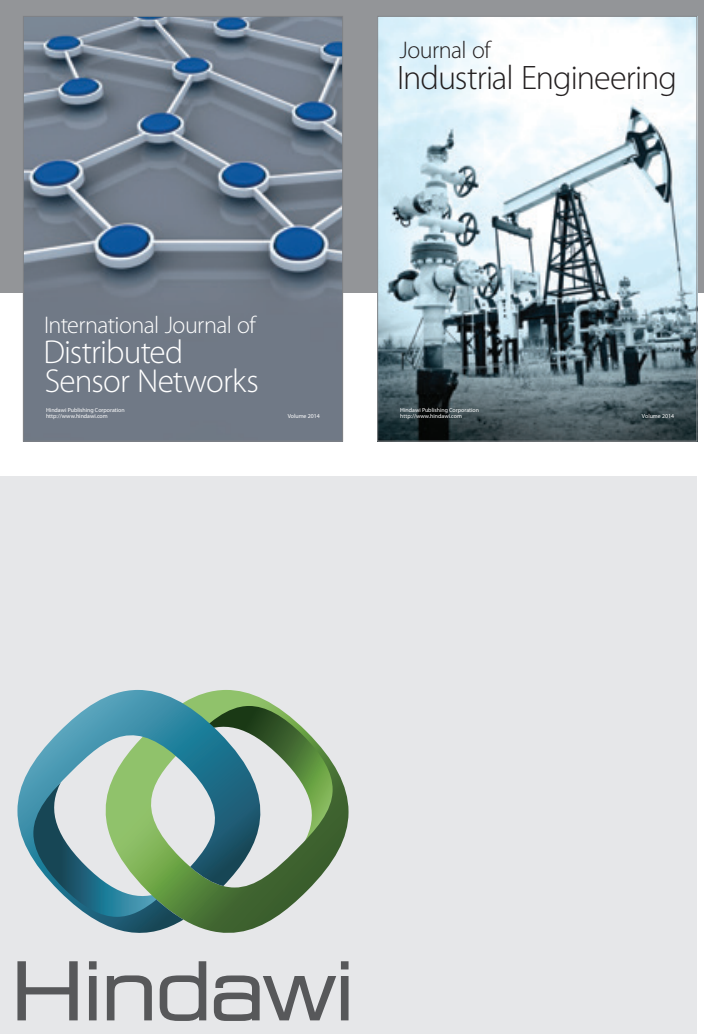

Submit your manuscripts at

http://www.hindawi.com

\section{Computer Networks} and Communications
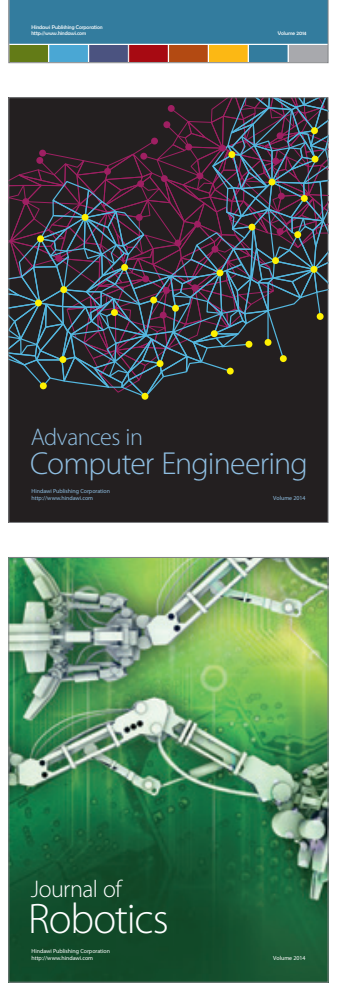
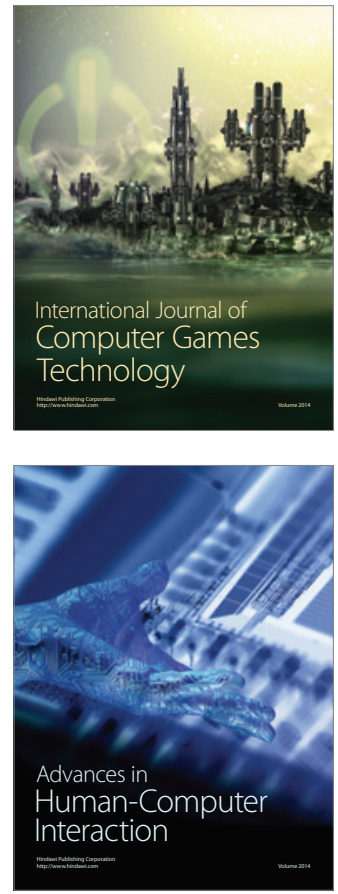
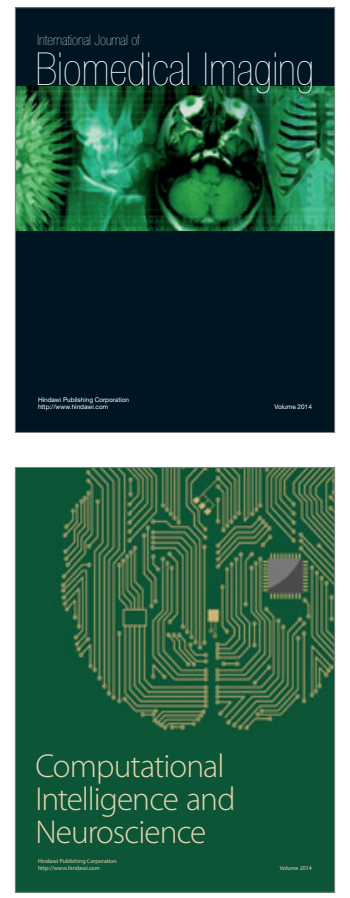
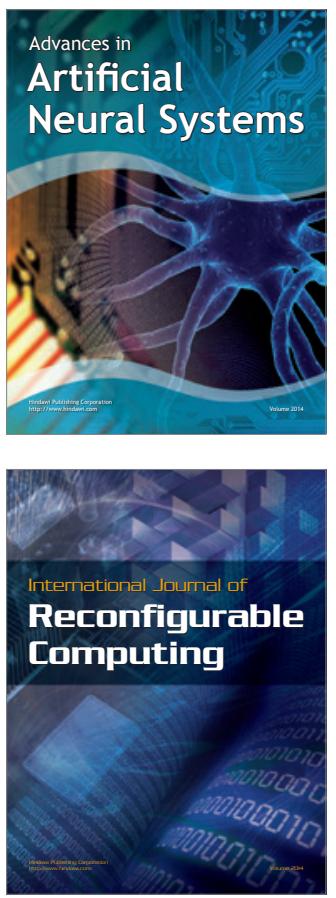
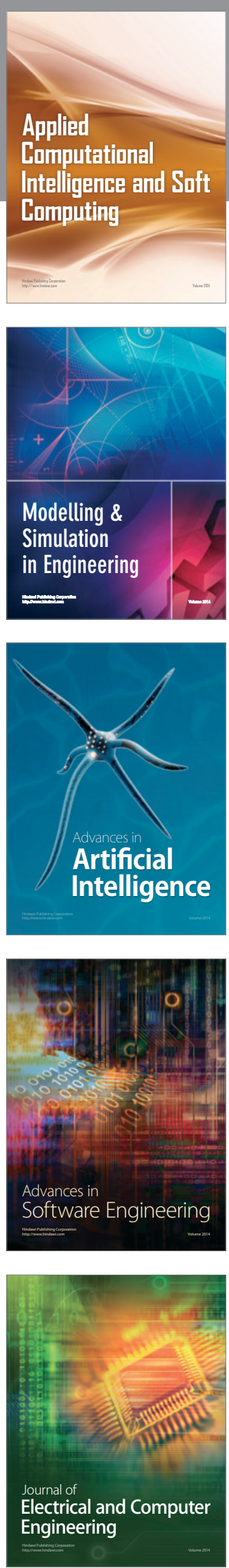